\title{
Fine structure of NQR spectra in GaSe
}

\author{
Z.D. Kovalyuk ${ }^{1}$, G.I. Lastivka ${ }^{2}$, O.G. Khandozhko ${ }^{2}$ \\ ${ }^{1}$ Chernivtsi Department of the Institute of Materials Science Problems, NAS of Ukraine, \\ 5, Iryna Vilde str., 58001 Chernivtsi, Ukraine, \\ Phone:(0372)52-51-55,fax (0372) 23-60-18; e-mail: chimsp@ukrpost.ua \\ ${ }^{2} Y u$. Fed'kovych Chernivtsi National University, \\ 2, Kotsyubyns'ky str., 58012 Chernivtsi, Ukraine, \\ Phone:(0372)24-24-36, fax (0372) 24-24-36; e-mail: rt-dpt@chnu.cv.ua
}

\begin{abstract}
Peculiarities of the observation of nuclear quadrupole resonance (NQR) in GaSe crystals grown from melt are under investigations. The splitting of a resonance NQR line by two identical spectra is caused by the availability of non-equivalent positions of $\mathrm{Ga}$ atoms in the polytypes $\varepsilon, \gamma$ and, probably, $\delta$. The appearance of a fine structure in NQR spectra is related to ordering in the system of the lattice defects (polytypes), when the atomic Se-Ga-Ga-Se layers are stacked during the crystal growth.
\end{abstract}

Keywords: nuclear quadrupole resonance, layer semiconductor, fine structure of spectra, polytype.

Manuscript received 27.05.09; accepted for publication 10.09.09; published online 30.10.09.

\section{Introduction}

Gallium selenide belongs to the group of layered semiconductor materials which attract active interest of researchers not only due to peculiarities of physical properties caused by strong anisotropy of the chemical bonds, but also because of a possibility of wide practical applications, as for instance, in photoelectronics [1,2]. The crystal structure of GaSe is arranged in such a way that the bonds within a separate Se-Ga-Ga-Se layer are essentially covalent, whereas those between the layers are of Van der Waals type. A weak bonding between the layers leads to their different stacking at formation of bulk crystals. A comparatively small energy difference of the formation of the different structure polytypes leads to the stacking faults between them that are structural defects typical for the layered crystals.

The compound GaSe may be crystallized into four polytypes: $\beta, \varepsilon, \gamma$, and $\delta$. The formation of a certain polytype, or their mixture, essentially depends on the method of obtaining these single crystals $[3,4]$. It is known that GaSe single crystals grown from melt (the methods of Bridgman or Czochralski) consist preferably of the $\varepsilon$-polytype at a low content of the $\gamma$ - and, possibly, $\delta$-modification. It was found in [5] that in such crystals the presence of the $\beta$-modification is hardly probable. The polytypes essentially affect the distribution of electron charge density and the formation of the band structure [6] what should be taken into account at development of optical spectrum devices. In this context, studies of structure defects in layered semiconductors remain a problem of today.

In this paper, it is shown that the method of nuclear quadrupole resonance (NQR) enables to identify polytypes in III-VI compounds of the GaS type. Additionally, the test-sensitivity to local environment gives a possibility to estimate the structure imperfection and to observe ordering in the system of defects. As the formation of structure defects appreciably depends on conditions of crystal growth and subsequent annealing, the NQR method is very convenient and, besides, nondestructive method for testing the quality of obtained crystals. Its efficiency is confirmed by observation of the multiplet structure in the resonance spectra caused by non-equivalent positions of $\mathrm{Ga}$ atoms due to the presence of polytypic modifications in GaSe crystals.

\section{Experimental}

GaSe single crystals grown by the Bridgman method have been used in experimental studies. The obtained samples had the $p$-type conductivity with a hole density of the order of $10^{15} \mathrm{~cm}^{-3}$. A comparatively high resistivity $\left(\sim 10^{6} \mathrm{Ohm} \cdot \mathrm{cm}\right.$ at $\left.T=293 \mathrm{~K}\right)$ results in reduction of the skin effect at the resonance frequency. This favours successful observation of NQR in largesize bulk samples. The latter circumstance makes it possible to test as-grown GaSe ingots and estimate their 
quality from the form of NQR spectra. The minimum volume of the samples necessary to have sufficient intensity of NQR signal is 0.5 to $1 \mathrm{~cm}^{3}$.

Investigations of NQR spectra was carried out by means of a spectrometer at continuous-wave operation in the radio-frequency bandwidth 19 to $21 \mathrm{MHz}$ by using Zeeman modulation, i.e. a periodic suppression of a NQR signal with magnetic field. In this case, a bipolar form of pulse magnetic field was used. Its amplitude was established experimentally and can achieve the value $100 \mathrm{Gs}$. In comparison to a frequency modulation, a Zeeman modulation gives best results for quadrupole nuclei located in surrounding with axial symmetry. Just such a situation takes place for $\mathrm{Ga}$ and In nuclei in the layered compounds of the GaS type. A time variation of magnetic field at the sample during passing the resonance conditions is shown in Fig. 1a and receiving a signal harmonic - in Fig. 1b. During the action of a magnetic field $+H$ or $-H$ (time ranges $t_{1}-t_{2}$ and $t_{3}-t_{4}$ ), the NQR signal is suppressed due to splitting the quadrupole energy levels and appears when the field is absent (time ranges $t_{0}-t_{1}$ and $t_{2}-t_{3}$ ). It is seen that during one period of the field variation the NQR signal appears twice. By using the technique of locked-in detection, one can observe spectra derivatives, the order of which depends on the selection of signal harmonic.

A peculiarity of NQR in the layered crystals of the $\mathrm{GaS}$ type is orientation dependence of the resonance signal intensity on mutual orientation of vectors of the high-frequency field $\mathbf{H}_{1}$ and the crystallographic $\mathbf{c}$ axis. The maximum intensity is observed at the $\mathbf{H}_{1} \perp \mathbf{c}$
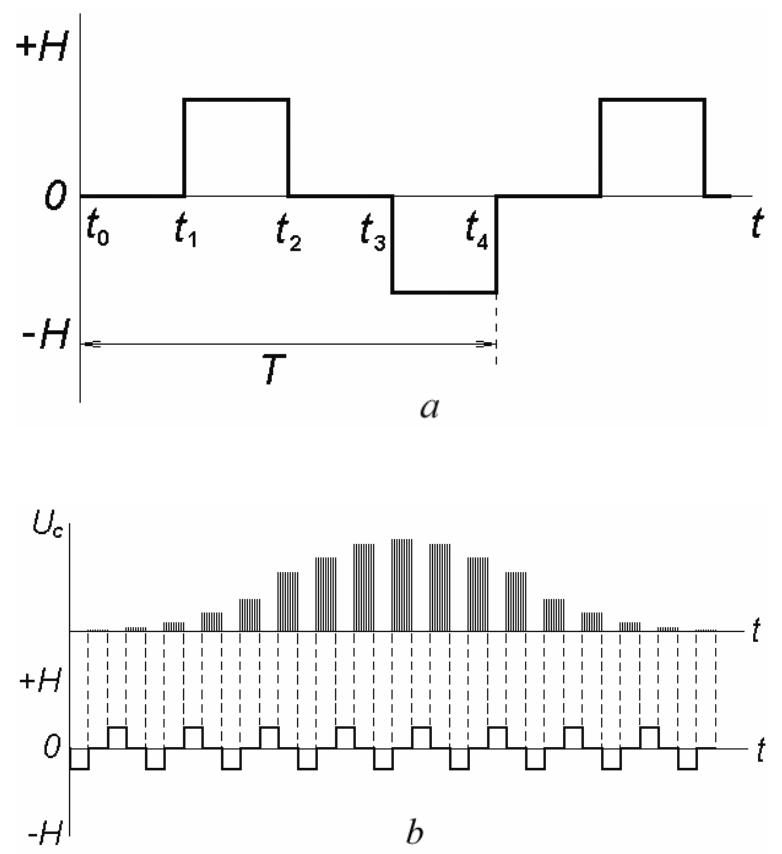

Fig. 1. The form of pulses of bipolar magnetic field applied to a sample $(a)$ and response signal at the output of a NQR spin-detector when using Zeeman modulation $(b)$. orientation and decreases to zero at $\mathbf{H}_{1} \| \mathbf{c}$. An analogous dependence was observed by us earlier for InSe [7] as well as by others authors in other compounds with layered crystal structure [8]. This confirms the anisotropy of the crystal structure and the existence of axial symmetry of the electric-field gradient in the local surroundings for $\mathrm{Ga}$ and In nuclei in GaSe and InSe compounds, respectively. The orientation of $\mathbf{H}_{1}$ and $\mathbf{c}$ vectors with respect to the layers of GaSe is shown in Fig. 2. It is this orientation that corresponds to the maximum intensity of NQR signals. The existence of orientation NQR dependence enables to detect a block structure of the single crystals.

Note that gallium has two resonance active isotopes $\left({ }^{69} \mathrm{Ga}\right.$ and $\left.{ }^{71} \mathrm{Ga}\right)$ with the quadrupole moments $e Q$ equal $0.2318 \cdot 10^{-24}$ and $0.1461 \cdot 10^{-24} \mathrm{~cm}^{2}$, respectively. As the ${ }^{69} \mathrm{Ga}$ isotope is more available in nature $(60.2 \%)$ in comparison to ${ }^{71} \mathrm{Ga}(39.8 \%)$ and appreciably higher quadrupole moment, NQR spectra are more intensive just for these nuclei. In our experiments, observation of NQR has been carried out preferably at ${ }^{69} \mathrm{Ga}$ nuclei.

\section{Results and discussion}

For ${ }^{69} \mathrm{Ga}$ and ${ }^{71} \mathrm{Ga}$ isotopes the nucleus spin $I=3 / 2$, therefore, according to the selection rules, in an electricfield gradient there is only one resonance transition $1 / 2 \pm 3 / 2$ for NQR. In this case, the resonance frequency $f$ between the levels of quadrupole energy can be determined from the relationship [9]:

$f=\frac{e Q g_{z z}}{2} \sqrt{1+\frac{\eta^{2}}{3}}$,

where $g_{z z}$ is the electric-field gradient in the sites of the gallium atoms and $\eta$ is the parameter of asymmetry that shows the deviation scope of the electric-field gradient of axially-symmetrical distribution. As GaSe belongs to the hexagonal system, the electric-field gradient is directed along the crystallographic $\mathbf{c}$ axis, i.e. along a Ga-Ga bond, and has axial distribution. It is confirmed

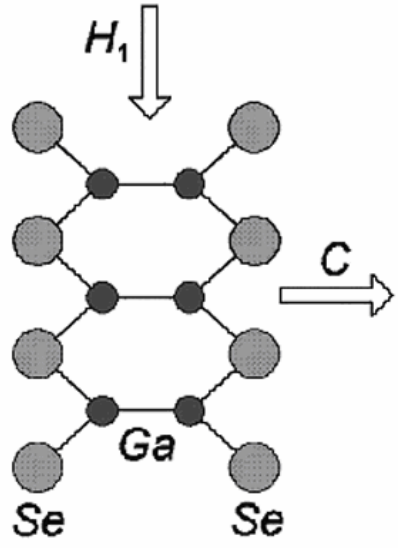

Fig. 2. The location of $\mathbf{H}_{1}$ and $\mathbf{c}$ vectors with respect to the layers of the crystal. 
by many investigations including those of EPR spectra [10]. Thus, in expression (1) the parameter of asymmetry may be neglected. From this viewpoint, under investigation of hypothetical GaSe sample with homogeneous crystal structure only a singlet NQR spectrum (one resonance line) should be observed. However, the registered spectra are multiplet. Fig. 3 shows a typical NQR spectrum obtained by the method of Zeeman modulation at ${ }^{69} \mathrm{Ga}$ nuclei of a GaSe single crystal.

In order to eliminate possible influence of conventional lattice defects appearing at growing single crystals such as twinning, mosaic structure or the presence of other phases on NQR spectra, we have registered X-ray topograms. It was found that at cleaved surfaces of GaSe samples the dislocation density did not exceed $2.5 \cdot 10^{3} \mathrm{~cm}^{-2}$. A well-defined separation of the $K_{\alpha 1}$ and $K_{\alpha 2}$ lines at the topograms obtained by the BergBarrett method is a confirmation of high quality of the single crystals. The Laue patterns with the absence of additional reflections, obtained in a polychromatic radiation of tungsten, reflect the hexagonal symmetry of the crystal. From an analysis of the X-ray data, it follows that there are no such lattice imperfections or additional phases able to cause the splitting of the NQR line by two doublets (Fig. 3). The appearance of the multiplet spectrum ought to be related with variation of the local crystal field due to the lattice polytypes. A similar spectrum for polycrystalline GaSe in paper [11] was explained by the presence of the polytypes. Later, we had observed two doublets in the NQR ${ }^{69} \mathrm{Ga}$ spectra in the single crystal samples and even in intercalated ones. Considering a possible reason for the appearance of two doublets it should be noted that the crystal lattice of GaSe grown by the Bridgman method usually is a mixture of the $\varepsilon$ - and $\gamma$-polytypes [1-3]. A peculiarity of these modifications is the availability of two groups of

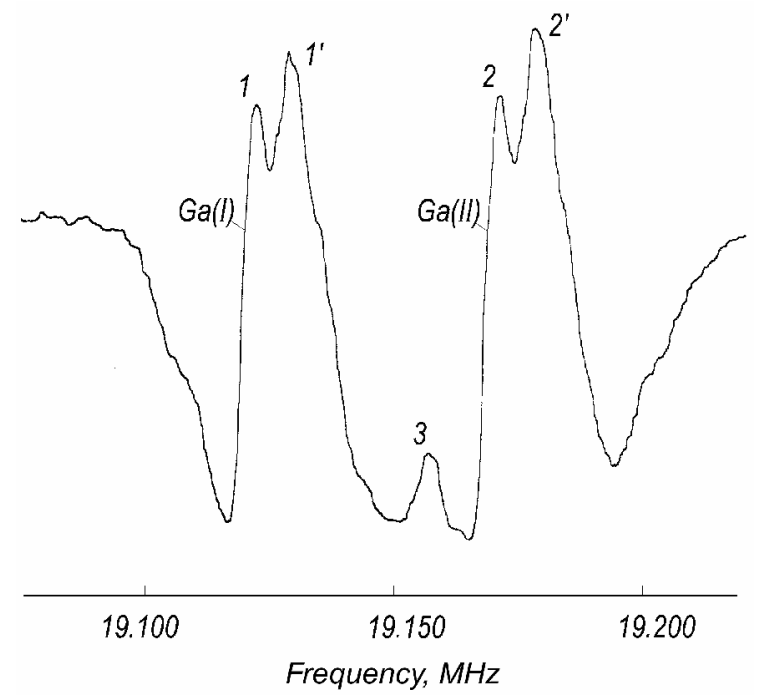

Fig. 3. $\mathrm{NQR}{ }^{69} \mathrm{Ga}$ spectrum in GaSe obtained by the method of Zeeman modulation at continuous passing the resonance conditions and synchronous detection of the second signal harmonic. $T=293 \mathrm{~K}$.
Ga atoms differing with their local surroundings. This is well seen in Fig. 4 where the crystal structure of the $\beta$-, $\varepsilon-, \gamma$-, and $\delta$-polytypes typical for $\mathrm{GaS}, \mathrm{GaSe}$, and $\mathrm{InSe}$ crystals is shown. If for the first group along the Ga-Ga bond direction all $\mathrm{Ga}$ atoms of some layer are opposite to Se atoms of an adjacent layer, then in the second group they are opposite to interstitials. Thus, two fourfold layers of the $\varepsilon$-polytype become non-equivalent with respect to each other due to redistribution of electron density at gallium atoms. This causes variation of the electric-field gradient at the gallium nuclei. Evidently, a similar situation takes place for the $\gamma$ - and $\delta$ polytypes (Fig. 4), too. Note that for the $\beta$-modification, always available in GaS, all the Ga atoms in the unit cell are equivalent [6] and the NQR spectrum contains only one slightly splitted line [11].

Thus, the two non-separated doublets in the NQR ${ }^{69} \mathrm{Ga}$ spectrum of GaSe belong to $\mathrm{Ga}$ atoms, which are located in two non-equivalent positions (designated as $\mathrm{Ga}(I)$ and $\mathrm{Ga}(I I))$. An important argument in favour of this conclusion is the same intensity of the both spectra as the amount of $\mathrm{Ga}$ atoms in these non-equivalent positions is equal. The difference in the surroundings of the gallium nuclei in the polytypes results in a splitting of the NQR line by two identical spectra frequencyshifted by $50 \mathrm{kHz}$. It confirms the presence of the polytypes $\varepsilon, \gamma$ and, probably, $\delta$ in the GaSe sample under investigation. Note that for the $\gamma$-polytype stacking the layers is analogous to the $\varepsilon$-modification (Fig. 4). A stacking distinction between the polytypes appears only after the second layer [3]. In this relation, considering possible contributions to the electric-field gradient in the first two layers as well as in two next ones, it is arguable that resonance frequencies for the $\varepsilon$ - and $\gamma$-modifications will be the same. Therefore, the NQR ${ }^{69} \mathrm{Ga}$ lines corresponding to these polytypes should coincide. However, the existing, although insignificant, differences in stacking the layers, interatomic distances, and lattice parameters for the different modifications (Table) affect the magnitude and distribution of the crystal field and, therefore, some variation of the electric-field gradient at the gallium nuclei in the $\varepsilon$ - and $\gamma$-polytypes. Evidently, essential influence on the magnitude of the electric-field gradient is caused by the interatomic distance between the Ga atoms coupled with covalent $p$-electron bonds. Assuming an increase of the electron density at gallium atoms with decreasing the interatomic distance, one can say about an increase of the electric-field gradient $q_{z z}$ at the Ga nuclei in the $\varepsilon$ polytype in comparison to the $\gamma$-polytype. In this case, the NQR frequency for the $\varepsilon$-polytype should be some higher than that for the $\gamma$-modification. Then, the resonance peaks of the unresolved doublets $1^{\prime}$ and $2^{\prime}$ (Fig. 3) may be attributed to the $\varepsilon$-modification and more low-frequency peaks 1 and 2 - to the $\gamma$-modification. However, our assumptions is not unambiguous as except for the data listed in Table there are other data some different from them. 
Table. Lattice parameters and interatomic distances for GaSe polytypes [12].

\begin{tabular}{|l|c|c|c|c|}
\hline \multicolumn{1}{|c|}{ Polytype } & $\beta$ & $\varepsilon$ & $\gamma$ & $\delta$ \\
\hline Lattice & & & & \\
parameters, A: $a$ & 3.755 & 3.755 & 3.739 & 3.755 \\
& 15.95 & 15.996 & 23.862 & 31.99 \\
\cline { 2 - 5 } $\begin{array}{l}\text { Se-Ga, within a } \\
\text { layer }\end{array}$ & 2.515 & 2.485 & 2.467 & 2.463 \\
\cline { 2 - 5 } $\begin{array}{l}\text { Ga-Ga, within a } \\
\text { layer }\end{array}$ & 2.391 & 2.383 & 2.386 & 2.457 \\
$\begin{array}{l}\text { Se-Se, within a } \\
\text { layer }\end{array}$ & 4.491 & 4.766 & 2.722 & 4.784 \\
\cline { 2 - 5 } $\begin{array}{l}\text { Se-Se, between } \\
\text { the layers }\end{array}$ & 4.199 & 3.840 & 8.847 & 3.890 \\
\cline { 2 - 5 } & & & & \\
\hline
\end{tabular}

Thus, it is a way to explain the splitting of the $\mathrm{Ga}(I)$ и $\mathrm{Ga}(I I)$ lines by doublets due to the shift of the resonance frequency for each modification. One can see from the spectrum that such the frequency shift due to the non-equivalent $\mathrm{Ga}$ positions in the both polytypes takes approximately the same value.

The use of Zeeman modulation, when applying a pulse magnetic field to the sample, leads to inhomogeneous broadening of NQR lines. As a result, the resonance spectra shown in Fig. 5 do not show a complex doublet structure. In order to improve the spectrometer resolution in the course of registering the spectra, a frequency modulation was applied.

Fig. 5 shows the spectrum obtained when passing the doublet located at higher frequencies (curve $\mathrm{Ga}(I I)$ in Fig. 3). A similar fine NQR spectrum was also observed at passing the $\mathrm{Ga}(I)$ doublet situated at lower frequencies. Curve 1 registered at a deviation of modulation frequency $\Delta f=5 \mathrm{kHz}$ indicates complexity of each doublet. The spectrum becomes still more complicated when we had used a lower modulation depth $\Delta f=1 \mathrm{kHz}$ (curve 2). As these spectra are equally reflected in both doublets, one can state with confidence that the resonance lines belong to the structures of the $\varepsilon-$, $\gamma$-, and $\delta$-polytypes. The appearance of the fine structure of the NQR spectra indicates the absence of lattice defects able to distort considerably the GaSe crystal field. It is necessary to point out a small width of some resonance lines $(\Delta v \sim 1 \mathrm{kHz})$. Such a width is typical for dipole broadening of a NQR line observed for rigid lattices in solids [9].

The most frequent reason for broadening NQR lines is lattice imperfection caused by internal stresses leading to distortions in mutual arrangements of atoms and, therefore, their electrical fields. However, starting from the observed line width and fine structure of the spectrum one can conclude that there is a small amount of conventional defects in GaSe samples. In fact, it is confirmed by the data of X-ray topography, as it was noticed above.

The question arises what is a reason for the complex structure of the $\mathrm{Ga}(I)$ and $\mathrm{Ga}(I I)$ doublets. The fine structure of the NQR spectra appears in the cases when in the sample there are non-equivalent nuclei being described by different values of the parameters $q_{z z}$. In this respect, there is some analogy to a fine structure of nuclear magnetic resonance (NMR) spectra caused by chemical shift. However, in NMR spectra the frequency shifts are caused by magnetic screening in constant magnetic field whereas in NQR spectra they are due to inequality of crystal's properties in the points where non-equivalent nuclei are located. It is natural to suppose that the fine splitting of the $\mathrm{Ga}(I)$ and $\mathrm{Ga}(I I)$ doublets is related not to local surroundings of $\mathrm{Ga}$ atoms but rather to properties of the polytypes, namely differences in interatomic distances, unit cell dimensions, and stacking

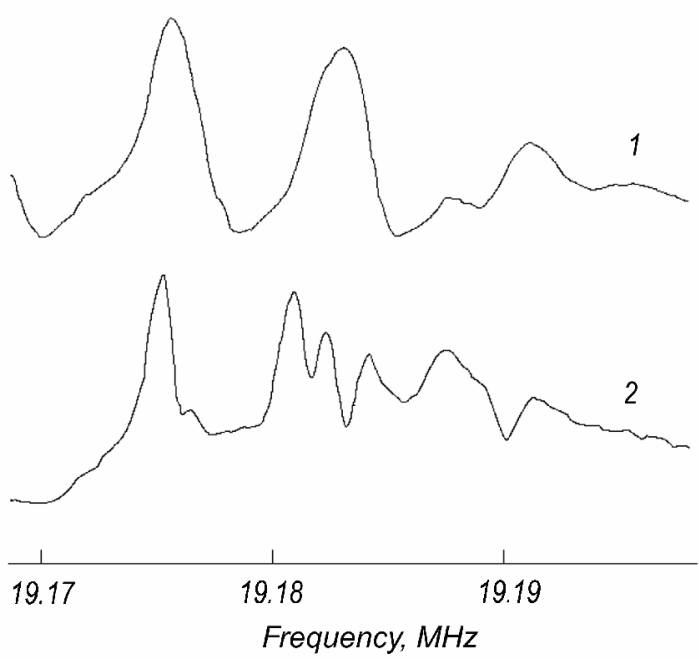

Fig. 5. Fine structure of the high-frequency doublet in the NQR spectrum shown in Fig. 3 for $\mathrm{Ga}(I I)$ for deviations of the modulation frequency $\Delta f=5 \mathrm{kHz}(1)$ and $1 \mathrm{kHz}(2)$.
Fig. 4. Polytypes of GaSe. Small filled circles - Ga atoms and large open circles - Se atoms. Unit cells are marked with rectangles [4].

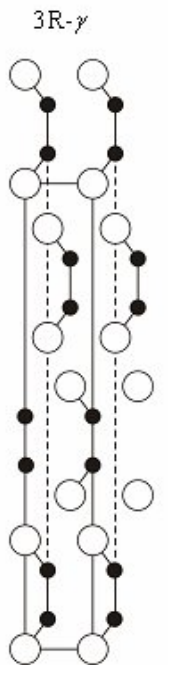

Physics, National Academy of Sciences of Ukraine 
order. The last circumstance, apparently, is decisive in the appearance of the fine NQR spectra. An ordered alternation of the polytypes with similar crystal symmetry and not too different interatomic Ga-Ga distances leads to a regular, incommensurate to the lattice, variation of electrical fields in the crystal. On contrary to a random alternation of the polytypes or their mixture, their ordered stacking along the $\mathbf{c}$ axis results to the appearance of a fine structure of the NQR spectra with narrow lines in GaSe. Thus, the observed fine structure and enough narrow lines of the NQR spectra in GaSe suppose the presence within the crystal the ranges with a high degree of ordering in the system of the polytypes. Unfortunately, the available discrepancy in the values of the known crystal structure parameters for the polytypes does not enable yet to relate definitely the observed spectral lines to a certain modification. We can only state that the observed fine structure of layered GaSe crystals is related to their perfection that is in dependence on the conditions of growing crystals and their subsequent treatment. Note that the samples under investigations were obtained from an ingot grown by the Bridgman method at a relatively high temperature gradient $\left(40{ }^{\circ} \mathrm{C}\right)$ at the crystallization front. For a single crystal obtained at a smaller temperature gradient $\left(20{ }^{\circ} \mathrm{C}\right)$ more broadened NQR lines were registered and any fine structure was not found. A similar situation took place in the NQR ${ }^{115}$ In spectra for InSe single crystals grown at the same conditions [13]. It enables to conclude that at crystallization in the field of a lower temperature gradient there is an increase of disordering in the system of structure defects in the layered crystal.

\section{Conclusion}

The carried out investigations of NQR spectra in the frequency range 19 to $21 \mathrm{MHz}$ confirm that, for the crystal structure of the layered compound GaSe, the axial symmetry of the electric-field gradient directed along a Ga-Ga bond is inherent. It results in a dependence of the NQR spectra intensity on mutual orientation of the crystallographic $\mathbf{c}$ axis and the magnetic component of the vector $\mathbf{H}_{1}$ for applied highfrequency field. The existence of this dependence makes it possible to detect disorientated blocks in single crystals. The non-equivalent positions of $\mathrm{Ga}$ atoms in the $\varepsilon$ - and $\gamma$-polytypes cause a splitting of NQR spectra by two identical spectral doublets separated in frequency by about of $50 \mathrm{kHz}$ at $T=293 \mathrm{~K}$. At a low level of frequency modulation of the resonance $(\Delta f<5 \mathrm{kHz})$, there is a fine spectrum in each doublet, which consists of a set of narrow lines. The appearance of the fine spectrum can say about ordered stacking of the polytypes $\varepsilon$ and $\gamma$ as well as polytypes of higher orders, which leads to a regularity in the change of the crystal field in GaSe along the c axis. It is the reason for the additional splitting of the resonance peak by separate lines with the dipole width. Thus, starting from the form of NQR spectrum in GaSe, it is possible to detect the presence of the polytypes $\varepsilon$ and $\gamma$, their relative content as well as to estimate quality of a layered sample under investigation.

\section{References}

1. K.A. Askerov, Prospective photodetectors for visible and near-IR spectral range // XVI Int. Sci. and Techn. Conference on Photoelectronics and Night Viewing Devices. May 25-27, 2000, Moscow, Part IV, p. SB13.

2. Z.D. Kovalyuk, O.A. Politanskaya, O.N. Sidor, V.T. Maslyuk, Electrical and photoelectric characteristics of structures based on layered InSe and GaSe semiconductors under irradiation with electrons of energy $12.5 \mathrm{MeV} / /$ Fizika Tekhnika Poluprovodnikov 42(11), p. 1321-1326 (2008) (in Russian).

3. M.S. Brodin, I.V. Blonsky, Excitonic Processes in Layered Crystals. Naukova Dumka, Kiev 1986 (in Russian).

4. J.C.J.M. Terhell, Polytypism in the III-VI layer compounds // Progr. Cryst. Growth and Characterization of Polytype Struct. 7, p. 55-110 (1983).

5. A. Baidulaeva, Z.K. Vlasenko, A. Dauletmuratov, L.F. Kuzan, P.Ye. Mozol', Raman spectra for GaSe single crystals subjected to influence of laser radiation // Fizika Tekhnika Poluprovodnikov 39(4), p. 405-408 (2005) (in Russian).

6. Y. Depeursinge, A. Baldereschi, Polytypism and layer-layer interaction in the III-VI layer semiconductor // Physica B 105 (1-4), p. 324-328 (1981).

7. V.V. Brailovskyy, G.I. Lastivka, O.E. Ilarionov, O.G. Khandozhko, Orientation dependence of NQR in InSe // Bulletin of Uzhgorod State University, Fizyka No. 21, p. 104-108 (2007) (in Ukrainian).

8. S. Levy, A. Keren, Pure nuclear quadrupole resonance determination of the electric field gradient asymmetry for broad lines // J. Mag. Resonance 167(2), p. 317-321 (2004).

9. V.S. Grechishkin, Nuclear Quadrupole Interactions in Solids. Nauka, Moscow, 1973 (in Russian).

10. V.I. Konovalov, S.S. Ishchenko, S.M. Okulov, Axial electrical field and EPR in GaSe // Fizika Tverdogo Tela 22(6), p. 1842-1844 (1980) (in Russian).

11. T.J. Bastow, I.D. Cambell, H.J. Whitfeld, $A{ }^{69} \mathrm{Ga}$, ${ }^{115}$ In NQR study of polytypes of GaS, GaSe and InSe // Solid State Communs. 39(2), p. 307-311 (1981).

12. G.L. Belen'kii, E.Yu. Salaev, R.A. Suleimanov, Deformation phenomena in layered crystals // Uspekhi Fizich. Nauk 155(1), p. 89-127 (1988) (in Russian).

13. Z.D. Kovalyuk, Ye.I. Slyn'ko, O.G. Khandozhko, Nuclear quadrupole resonance in polytype compounds GaSe and InSe // Fizyka $i$ Khimiya Tverdogo Tila 2(4), p. 579-583 (2001) (in Ukrainian). 